\title{
Mensuração de desempenho em organizações públicas: caso da Secretaria da Fazenda do Rio Grande do Sul
}

Vinicius Pimentel de Freitas, Ricardo Lopes Cardoso, André Carlos Busanelli de Aquino e Rômulo Campos dos Reis Júnior

\section{Introdução}

Este artigo apresenta estudo de caso da implantação de um modelo de mensuração de desempenho na administração pública brasileira. Como observado em outros países da América Latina e em outras economias em desenvolvimento, a gestão de entidades públicas no Brasil passa por um movimento de aprimoramento, que foi iniciado com a consolidação da democracia (no final dos anos 1980) e o controle do processo inflacionário (no final dos anos 1990).

Seja em organizações públicas ou privadas, modelos de mensuração de desempenho são utilizados como forma de promover alinhamento do comportamento individual à estratégia da organização (EPstein; Manzoni, 1997). Esse alinhamento é composto por melhoria da tomada de decisão e por geração de incentivos - na terminologia de Sprinkle (2003) -, facilitando e influenciando decisões, respectivamente. Tais modelos são compostos por indicadores de desempenho e relações de causa e efeito. Os indicadores sinalizam em que grau 
as diversas equipes e áreas estão aproximando-se de uma condição de desempenho desejada, nas mais diversas dimensões, por exemplo: montante arrecadado, fiscalizações executadas, autos de infração lavrados. Tais indicadores, geralmente associados entre si por meio de relações de causa e efeito, sugerem uma expectativa aos gestores de que, adotando-se curso de ação dentro das relações causais sugeridas, os indicadores finais serão alcançados com maior probabilidade e intensidade. Assim, essas associações causais entre decisões e resultados esperados dão suporte ao desenvolvimento, comunicação e execução das estratégias da organização (MALINA; Selto, 2004). Para alinhar as escolhas individuais de gerentes e demais participantes com os objetivos da organização, os indicadores são ligados ao processo orçamentário e sobre esses são estabelecidas metas, utilizadas não só para fins de decisão da fase de avaliação, mas também com a finalidade de gerar incentivos às equipes. Dessa forma, surge a tendência das equipes gerenciarem as metas, criando folgas orçamentárias, ou seja, estabelecendo metas mais fáceis de atingir e que possam acomodar incertezas geradas por contingências futuras (MERCHant, 1985). Tais metas podem ser estabelecidas de forma negociada ou imposta pela alta gerência. A participação na definição das metas acresce a motivação e o comprometimento das equipes, se comparada à condição de metas impostas pela supervisão (Brownell, 1982).

Outros efeitos que afetam o processo são a dispersão dos esquemas de pagamento e a força das relações causais. Esquemas de bonificação baseados em esforços da equipe dispersam incentivos, na medida em que dissociam esforço individual do resultado da equipe, a qual serve como base para o bônus (WIDENER, 2006). O comprometimento com a meta também depende do quanto os gestores confiam nas relações causais presentes nos indicadores, chamadas de "força das relações" (WEBB, 2004). Em estudo na estrutura de incentivos e orçamento de uma empresa de distribuição de energia elétrica, Aquino et al (2008) encontraram que a dispersão do esquema de pagamento modera o impacto da incerteza na criação de folga orçamentária, mas não encontraram o mesmo efeito para a força das relações causais.

$\mathrm{Na}$ iniciativa privada, essa forma de alinhamento é usada largamente e está intimamente ligada à administração contemporânea. O movimento do New Public Management, iniciado na década de 1980, defende a adesão das entidades públicas a uma maior orientação de mercado e busca por eficiência na gestão de custos. Nesse sentido a adoção de modelos de desempenho vem crescendo na administração pública em função da demanda de melhorias efetivas nos serviços públicos. Tais modelos parecem ter papel importante na promoção do alcance das metas de uma organização, o que depende da implantação e da comunicação da estratégia, além de um conjunto compreensível de medidas de desempenho ligadas à estratégia e aos resultados da organização (em uma relação de causalidade). É desejável que as medidas de desempenho sejam relacionadas às ações dos gerentes, que se configurem em metas desafiadoras (contudo atingíveis), e que sejam, ainda, relacionadas com recompensas significativas (BROWNELL, 1982; Malina; Selto, 2001; Malina; Selto, 2004). Espera-se que o uso efetivo desses modelos, mais que simples esforço de monitoramento, atue como gerador de incentivos a ações mais eficientes. 
Embora a adoção de modelos de mensuração de desempenho nas organizações públicas seja comum, muitos desafios ainda existem. Esses modelos estão sendo utilizados extensivamente para fornecer informações a gabinetes de planejamento e de orçamento, embora com poucos resultados práticos tanto em decisões estratégicas quanto para incentivo de projetos, o que é afetado por questões políticas (AmMOns; RivenBARK, 2008).

Estudos recentes vêm mostrando que têm mostrado que, em muitos países, esforços significativos têm sido feitos por diversas instâncias na administração pública para medir os resultados de suas decisões e diminuir a discricionariedade no processo orçamentário (CAIDEN, 1998). Entre esses esforços está o uso de indicadores de desempenho em associação com o orçamento, como ocorre nos Estados Unidos (Melkers; Willoughby, 2005), no Reino Unido (James, 2004) e atualmente no Brasil, tanto na esfera federal, como o uso do conceito de "mapa estratégico" pelo Tribunal de Contas da União, quanto nas esferas estadual e municipal. A partir de 2007, os estados de São Paulo, Pernambuco e Rio de Janeiro adotaram o uso de indicadores para acompanhamento das ações de reorganização de processos, revisão de despesas e coordenação de investimentos no estado, a exemplo das cidades de Porto Alegre, Curitiba e Sorocaba (PADUAn, 2009).

No sentido de compreender a adoção desses modelos em diversos países, devem ser consideradas as suas diferenças institucionais. $\mathrm{Na}$ literatura de contabilidade gerencial, estudos mostram que as métricas de mensuração de desempenho, assim como os mecanismos de coordenação empregados para geração de informação voltada à decisão e promoção de incentivos, sofrem a influência do ambiente institucional na sua adoção, adaptação e evolução (SCAPENS, 1994). Essa influência institucional pode vir das instituições formais (como a legislação de um país) ou mesmo das informais (como a cultura).

Neste estudo, analisamos a implantação de um modelo de desempenho na Secretaria de Estado da Fazenda do Rio Grande do Sul (Sefaz) ${ }^{1}$ e sua relação com a remuneração variável (prêmio de produtividade e eficiência) dos funcionários

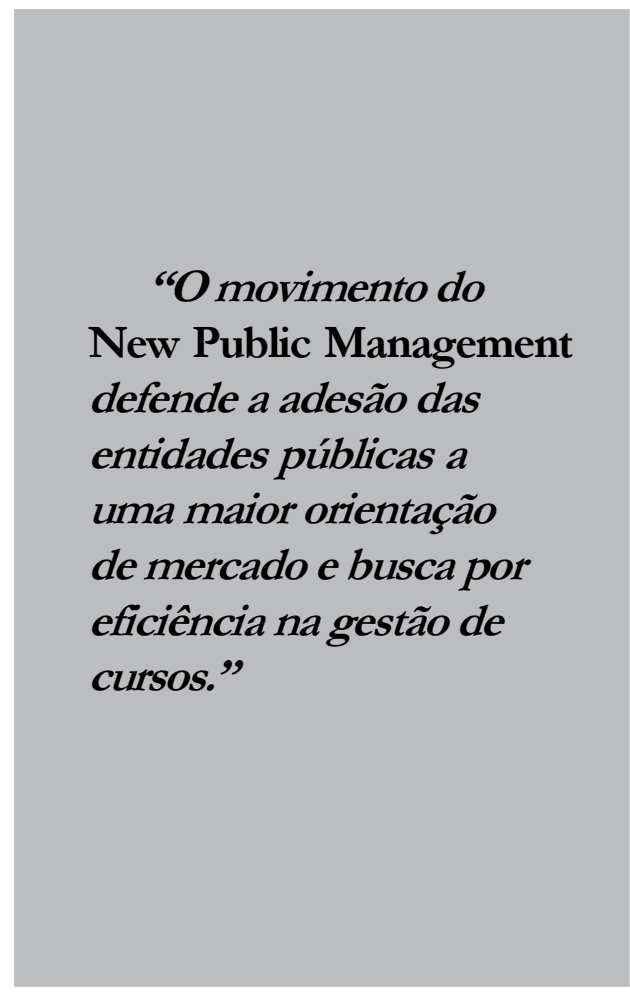

alocados nessa secretaria; a qual, segundo o Decreto Estadual 37.297 de 1997, é responsável pela arrecadação e fiscalização dos tributos estaduais e pelas funções tradicionais de contabilidade estadual, de controle interno e de gerenciamento das despesas.

O estudo de campo partiu de uma abordagem exploratória e descritiva, baseado em análise documental e 
entrevistas em profundidade com funcionários públicos lotados na Sefaz. A contribuição do uso de estudos de campo nessa fase da pesquisa está no ganho de riqueza de detalhes do fenômeno, gerando maior entendimento das práticas correntes (Humphrey; Scapens, 1996). Na condução das entrevistas, a triangulação de evidências foi feita em parte com o informante-chave, um dos autores, o qual trabalha na organização como agente fiscal desde 1994.

De forma breve, o modelo de desempenho da Sefaz começou a ser implantado em 1997, a partir de discussões sobre planejamento estratégico. Como uma resposta à falta de motivação dos funcionários, em 2003 foi iniciada a implantação do sistema de incentivos. Estes incentivos incluem prêmios por desempenho de equipes, eventuais promoções a cargos decisórios (e consequentes adicionais salariais) e reconhecimento da capacidade gerencial. A análise da série anualizada de indicadores consolidados dá evidências de que os funcionários da Sefaz têm atingido, em média, 90\% das metas estabelecidas. Isso sugere a existência de folgas orçamentárias e, portanto, de baixo incentivo à eficiência. Apesar dos resultados deste estudo não serem diretamente generalizáveis a outras organizações públicas, o estudo aponta que as variáveis relevantes à dinâmica organizacional, de decisão e incentivos em organizações privadas, também ocorrem em organizações públicas.

Além desta introdução, o presente artigo está estruturado da seguinte forma. Inicialmente é apresentada breve literatura sobre modelos de mensuração de desempenho para administração pública. Em seguida, descreve-se o modelo de mensuração de desempenho na Sefaz e são demonstradas as evidências coletadas no caso. Finalmente, são apresentadas as considerações finais, bem como sugestões de problemas para pesquisas futuras.

\section{Mensuração de desempenho na administração pública}

Da mesma forma que os estudos para organizações privadas, alguns estudos mostram que a administração pública tem a expectativa de que a remuneração variada afete o comprometimento dos funcionários e direcione escolhas. Melkers e Willoughby (2005) apresentam evidências de que muitos estados norte-americanos definiram prêmios (ou sanções) relacionados com o atingimento (ou não) de metas especificadas em seus modelos de desempenho, sendo os prêmios mais frequentes do que as sanções.

Tais prêmios, no caso dos estados norteamericanos, existem tanto no nível departamental quanto no nível individual. Em nível departamental os prêmios são distribuídos pelo reconhecimento do trabalho (em 27\% das cidades e $23 \%$ dos distritos) ${ }^{2}$ e pelo uso discricionário de recursos extras (em 13\% das cidades e dos distritos). Já no nível individual os prêmios contemplam cerimônias de premiação (em $23 \%$ das cidades e $22 \%$ dos distritos) e pagamento pecuniário individual pelo desempenho (em 17\% das cidades e $23 \%$ dos distritos). Essa divisão, parte dos prêmios associados a equipes e ao desempenho individual, também é encontrada na administração privada, a qual demonstra que quanto mais associado a equipes mais disperso é o incentivo gerado, por problemas de ruídos na identificação do esforço individual colocado no trabalho (WIDENER, 2006).

O estudo ainda identificou que o uso da informação de orçamento é baixo tanto no processo decisório (na redução de serviços 
ineficazes ou eliminação de serviços duplicados) quanto na melhoria da cooperação ou coordenação com o governo externo. Essas descobertas convergem para os resultados encontrados por Willoughby (2004), sugerindo que o orçamento está mais associado ao alinhamento das perspectivas dos agentes e à redução do risco moral do que ao processo decisório. Assim, o uso de indicadores de desempenho é mais relevante durante o fechamento do orçamento (ex ante) do que no uso para análise do desempenho efetivo (ex post) (MelKers; Willoughiby, 2005; Jordan; HaCKBart, 1999).

Em seu estudo, Willoughby (2004) também levantou importante aspecto que distingue a administração privada da pública; nesta última, o orçamento é influenciado por fatores políticos. De maneira similar, Bourdeaux (2006) demonstrou que o desenho institucional influencia a aplicação do orçamento, tanto na efetiva ação do Legislativo como agente do processo quanto no monitoramento orçamentário.

Ainda em relação ao uso dos indicadores de desempenho para decisão, James (2004), no caso do Serviço Público do Tesouro Britânico, identificou que, apesar de a função de monitoramento ter melhorado com o uso de indicadores de desempenho, estes criavam uma impressão limitada e desorientadora de análise do progresso, pois desviavam a atenção para as metas mais valorizadas no modelo. Resultados similares foram encontrados em experimentos com julgamento de avaliação de desempenho para administração privada (ITTNER et al., 2003).

\section{A gestão de desempenho na Sefaz}

O modelo atualmente em uso na Sefaz é resultado de três movimentos distintos: a implantação do processo formal de planejamento estratégico, a abertura institucional-legal para uso de remuneração variável na secretaria e a criação da carreira de Agentes Fiscais do Tesouro do Estado, que se unificou das duas antigas carreiras da Sefaz, ambas de nível superior.

As primeiras iniciativas de implementação de um planejamento estratégico estruturado na Secretaria datam de 1997, com a introdução do gerenciamento pela Qualidade Total. Consultorias externas introduziram no processo de gestão o uso de ferramentas e rotinas de qualidade, assim como o uso de metas. No mesmo ano, por meio de uma lei estadual, o governador foi autorizado a remunerar os funcionários ativos adicionalmente por meio de parcelas variáveis, pagas a cada funcionário segundo o desempenho da secretaria no trimestre, mas não incorporável ao salário (Sefaz, 2008). Essa remuneração, atualmente denominada de "Prêmio de Produtividade e Eficiência", foi implantada decorridos oito anos da sua permissão.

Em seguida, a nova administração da Sefaz, de 1999-2002, implantou, aliado ao já existente processo baseado em metas de qualidade total, o processo formal de planejamento estratégico baseado em indicadores. Finalmente, a equipe diretiva para o período 2003-2006, formada por 18 pessoas (incluindo secretário, secretário substituto, seus assessores, diretores de departamento e supervisores), ampliou o entendimento dos propósitos específicos que toda secretaria deveria perseguir. Nessa ocasião foram estabelecidos ao todo 32 propósitos específicos. Ao fazer isso, o modelo de planejamento migrou do tradicional foco de poucos indicadores para um modelo multi-indicadores (atualmente cerca de 180 indicadores distribuídos nos 32 propósitos citados), similar ao 
conceito de indicadores balanceados, financeiros e não financeiros. Essa diretoria considerou como motivos para a ampliação de indicadores a falta de motivação de funcionários, a implantação incompleta do ciclo gerencial, as dificuldades de aplicação do planejamento global às divisões e seções e a falta de comprometimento da média gerência. ${ }^{3}$ Para a implantação do processo de planejamento formal a diretoria intensificou treinamento em práticas de gestão para alta direção e gerência média, desenvolvimento e formação de equipes, formalização e padronização do processo de planejamento e introdução da remuneração variável - já sustentada institucionalmente (SEVERO, 2006). Hierarquicamente, a formalização da estrutura de planejamento foi feita pela criação do cargo "Assessor de Planejamento" para cada direção de departamento, estando esses assessores reunidos em um comitê interdepartamental, sob coordenação da Sudesq. ${ }^{4}$

O estabelecimento de metas no processo de planejamento é compartilhado e negociado entre supervisor e equipe, como tratado em Brownell (1982). O ciclo do planejamento segue o ano civil e é disparado pela equipe diretiva, que propõe propósitos específicos desdobrados dos propósitos gerais, estabelecidos pelo Governo do Estado e norteados pela Missão e Visão da Sefaz. A média e a baixa gerência fazem sugestões sobre ações específicas, as quais retornam para validação da equipe diretiva. Uma nova rodada é conduzida pelas média e baixa gerências, para que sejam definidos os objetivos, metas, ações e etapas que têm por finalidade prover os meios de alcançar cada propósito específico. Essas metas são validadas finalmente pela equipe diretiva.
O processo de planejamento é suportado por um sistema de informações. A atualização do status de consecução das metas em cada indicador é responsabilidade de cerca de 200 funcionários, dos 1.800 de carreira da secretaria. Cada indicador recebe uma avaliação do próprio funcionário responsável e do supervisor desse indicador. Caso a avaliação do grau de consecução da meta do indicador no período seja diferente entre a feita pelo funcionário responsável e seu supervisor, tal divergência fica apontada no sistema para ser analisada pela equipe diretiva. Os dados são agregados pelo sistema e utilizados pela equipe diretiva para acompanhar o desempenho geral da secretaria.

A atribuição mensal de pontos no sistema de premiação da Sefaz é proporcional ao alcance das metas dos 180 indicadores no mês. A remuneração variável dos funcionários da secretaria é uma parcela paga no trimestre, igual para todos os funcionários dentro de uma mesma classe funcional, a saber: Agentes Fiscais do Tesouro do Estado e Técnicos do Tesouro do Estado (Sefaz, 2008, art. 9-A). Essa remuneração é denominada "Prêmio Produtividade" e foi implantada em 2005.

O montante do prêmio a ser distribuído em parcelas iguais na classe funcional é uma porcentagem do montante da arrecadação estadual. Os agentes fiscais do Tesouro do Estado dividem um prêmio de 0,00000001665\% do montante total arrecadado, enquanto os técnicos do Tesouro do Estado dividem 0,00000000753\% do mesmo montante. Tais percentuais podem ser alterados pelo governador (e o são com frequência), com a finalidade de implantar planos fiscais especiais de governo.

Desde que a remuneração variável passou a ser associada ao planejamento estratégico, os funcionários percebem que 
o trabalho está mais focado e que os resultados estão melhores do que antes. Essa última observação é coerente com os dados apresentados por Melkers e Willoughby (2005, p. 188). Esse resultado pode ser efeito de maior atenção dirigida aos indicadores associados à pontuação para fins de remuneração variada ou de maior estruturação e melhor comunicação da estratégia global.

\section{Incentivos e folgas orçamentárias}

Entre os diversos tipos de incentivos presentes em organizações, os pecuniários de curto prazo e as promoções, por meio de mobilidade vertical ou horizontal, são os que mais afetam positivamente a ação de indivíduos (BesanKo, 2007). Evitar punições como demissão também afetaria o grau com que um funcionário coloca esforço, físico ou intelectual, em uma atividade. Contudo, esse último é menos efetivo para funcionários da administração pública pelas características de seu contrato de trabalho.

No caso da Sefaz, mobilidade vertical implica acréscimo salarial temporário por indicação a funções específicas, além do cargo ocupado. Assim, a probabilidade de ser recomendado a essas posições pode atuar como incentivo ao desempenho, na medida em que o agente deseja sinalizar competência.

Outro tipo de mobilidade, a horizontal, está mais associada à adequação de natureza de atividade ao desejo ou vocação particular do funcionário. Com base em entrevistas, não foi observado influência do prêmio de produtividade na mobilidade das pessoas entre as divisões da Sefaz.

Em termos de incentivo pecuniário, o prêmio representa aproximadamente 30\% dos vencimentos dos funcionários novos nas carreiras, e esse percentual cai para 10\% a $15 \%$ no caso de funcionários com mais de trinta anos de trabalho para o Estado e que possuem cargos de chefia. Assim, quanto maior o tempo de casa e a posição hierárquica, menor o incentivo da remuneração variável, em comparação com outros funcionários. A percepção dos funcionários da Sefaz é que a proporção atual entre o prêmio e outras parcelas de sua remuneração é adequada. Percentuais

"Alguns estudos

mostram que a

administração pública

tem a expectativa de

que a remuneração

variada afete $o$

comprometimento dos

funcionátios e direcione

escolhas."

mais altos de renda variável poderiam ser usados pelo governador como uma forma de capturar os técnicos da Sefaz, o que é coerente com o argumento apresentado por James (2004). Como o prêmio é dividido pela equipe, independentemente do nível de esforço do funcionário, o efeito redutor de incentivo pela dispersão do pagamento ocorre, pois há espaço para o efeito "carona" (Widener, 2006). 
Embora não exista mecanismo formal instituído de punição ao efeito "carona", as entrevistas apontam a existência de punição social aos funcionários e gerentes que puxam a média de consecução das metas para baixo; afinal, todos têm permissão de consulta aos indicadores de desempenho e seu responsável.

Por meio da observação das séries trimestrais dos indicadores de desempenho, na comparação meta/realizado entre 2005-2008 (14 trimestres, até junho/ 2008), observam-se evidências iniciais de que os funcionários da Sefaz têm atingido em média 91,46\% das metas (Tabela 1).

Percebe-se uma oscilação no atingimento das metas ao longo do trimestre no mesmo ano. As metas são estabelecidas no último trimestre de cada ano, para vigência a partir de janeiro do ano civil subsequente e, a priori, não devem ser ajustadas, pelo desempenho corrente, ao longo do ano. Entretanto, eventualmente a equipe diretiva pode aprovar revisões de metas caso eventos exógenos à secretaria induzam tal necessidade, como a recente crise financeira que reduziu a expectativa de arrecadação de ICMS.

A média gerência tem considerável influência na fixação das metas; algumas dessas podem ter sido progressivamente estabelecidas para serem de mais fácil atingimento (folga orçamentária). Contudo, a diferença entre o desempenho alcançado e a meta pode ser explicada por outros efeitos, além da folga orçamentária intencionalmente criada. Por exemplo, os funcionários se tornam mais habituados aos processos de planejamento estratégico e ao modelo de desempenho; assim suas ações se direcionam mais diretamente ao trabalho a eles relacionado, e menos esforço é desperdiçado em tarefas que não são relevantes para os objetivos da organização - curva de aprendizado associada à eficiência operacional.

\section{Considerações finais}

A presente análise exploratória sugere que o modelo de mensuração de desempenho da Sefaz contém algumas das características encontradas também na aplicação de tais modelos na iniciativa privada. Exemplos dessas similaridades são: dissociação de recompensa e esforço; negociação de metas como forma de comprometimento; evidências iniciais de folga orçamentária; e mecanismos sociais de punição adicionais aos incentivos

\section{Tabela 1: Percentagem de alcance de metas desde 2005, por trimestre, e} Estatística Descritiva

\begin{tabular}{l|r|r|r|l|c}
\hline Trimestre & Porcentual & \multicolumn{1}{|c|}{ Trimestre } & Porcentual & \multicolumn{2}{|c}{ Estatistica Descritiva } \\
\hline $20051 \mathrm{~T}$ & 93,8 & $20064 \mathrm{~T}$ & 87,91 & $\mathrm{n}$ & 14 \\
$20052 \mathrm{~T}$ & 87,27 & $20071 \mathrm{~T}$ & 84,78 & Média & 91,456429 \\
$20053 \mathrm{~T}$ & 81,01 & $20072 \mathrm{~T}$ & 95,49 & Mediana & 92,47 \\
$20054 \mathrm{~T}$ & 87,42 & $20073 \mathrm{~T}$ & 96,56 & Variância da amostra & 28,644717 \\
$20061 \mathrm{~T}$ & 95,35 & $20074 \mathrm{~T}$ & 97,42 & Desvio padrão & 5,352076 \\
$20062 \mathrm{~T}$ & 88,29 & $20081 \mathrm{~T}$ & 95,81 & Curtose & $-0,925138$ \\
$20063 \mathrm{~T}$ & 91,14 & $20082 \mathrm{~T}$ & 98,14 & Assimetria & $-0,462157$ \\
\hline
\end{tabular}

Fonte: Sisplan/Sefaz 
pecuniários. Primeiro, o potencial efeito "caronista" presente no esquema de pagamento de bônus decorre da dissociação do esforço individual realizado pelo funcionário e da quantia recebida por ele. Esse efeito é moderado pela punição social, pois os demais funcionários têm acesso de consulta ao sistema que individualiza o desempenho efetivo.

Apesar de a meta ser proposta pelo grupo de funcionários que a executa e validada pela equipe diretiva, essa não pode ser revisada ao longo do ano, o que poderia gerar incerteza. Estudos apontam que a incerteza tem um efeito direto no uso de folgas orçamentárias, por meio das quais os funcionários se protegem de contingências indesejáveis (AQuino et al, 2008). No caso em questão, os funcionários sabem que quando a probabilidade de alcance de uma meta é significativamente afetada por variáveis exógenas, a equipe diretiva tende a aceitar a revisão da meta. Esse comportamento da direção reduziria o impacto da incerteza na geração de folgas no orçamento.

Diferentemente de uma organização privada, o efeito "carona" na instância pública é teoricamente potencializado pela estabilidade no emprego. Outra diferença é o impacto do revezamento de cargos entre supervisor e subordinados no relaxamento de medidas punitivas. Por fim, comparado às iniciativas de modelos de mensuração utilizados na iniciativa privada contemporânea, o sistema da Sefaz não contempla relações explícitas de causa e efeito entre indicadores de desempenho. Assim, a não formalização de um mapa estratégico gera ausência de informação de causalidade, o que também pode ter efeito na criação de folga (WEBB, 2004), na medida em que a falta de informação gera incerteza sobre o comportamento do indicador e, consequentemente, o esforço que deveria ser empregado em tal indicador.

Como sugestão de pesquisa futura, a natureza dos indicadores poderia ser investigada. Entre os cerca de 180 indicadores de desempenho presentes nos 32 propósitos específicos, alguns são de natureza mais subjetiva, enquanto outros de natureza mais objetiva. Estes últimos reduzem a ambiguidade na interpretação por parte dos gerentes e geralmente são de natureza contábil-financeira (HARTMANN, 2000). A ênfase dada a indicadores de natureza contábil pode incentivar o uso de folgas orçamentárias. Enquanto a vaguidade presente nos de natureza subjetiva pode propiciar a criação tanto de folgas orçamentárias quanto de punições não cabíveis. Assim, para aprofundamento do estudo, sugere-se a análise da natureza dos indicadores que compõem o modelo. Pesquisas futuras também poderão analisar a dinâmica do estabelecimento de metas relacionadas à eficiência de diversos serviços públicos, como educação, ou mesmo à eficiência no uso de ativos. Recentes casos de sucesso no aprimoramento da gestão pública brasileira, relatados, por exemplo, por Paduan (2009), englobam as medidas estabelecidas pela Secretaria de Educação de Pernambuco para avaliar e recompensar professores da rede estadual, e as medidas estabelecidas pela Secretaria de Segurança Pública do Rio de Janeiro para apoiar a decisão de terceirizar a manutenção da frota, aumentando a taxa de uso desses ativos. Casos como esses poderão suscitar aperfeiçoamentos das medidas já adotadas, bem como a disseminação de práticas que poderão ser mimetizadas por outros setores e agentes da administração pública nacional. 
Por fim, apesar de os resultados não serem diretamente generalizáveis a outras organizações públicas, o estudo aponta que as variáveis relevantes à dinâmica organizacional, de decisão e incentivos, em organizações privadas também ocorrem em organizações públicas.

(Artigo recebido em abril de 2009. Versão final em setembro de 2009).

\section{Notas}

1 O Estado do Rio Grande do Sul é uma das 27 unidades da Federação brasileira, com 10,2 milhões de habitantes e PIB de US\$ 100 milhões em 2007 (FEE, 2008), o que corresponde ao quarto maior Estado brasileiro. O PIB brasileiro em 2007 é estimado em US\$ 1.300 Bilhões (Banco Central do Brasil, 2008).

2 Do texto original, traduzimos 'city' como 'cidade' e 'county' como 'distrito'.

3 Estas últimas são produto da alternância política no poder. Como as posições são ocupadas por funcionários de carreira, funcionários que assumem posições de gerência em uma administração podem (e muito provavelmente vão) perder autoridade em outra e vice-versa. Assim, observa-se uma tendência natural ao relaxamento de atitudes gerenciais.

4 A Sudesq, Supervisão de Desenvolvimento Organizacional e de Qualidade, é o órgão responsável pelos programas de desenvolvimento dos servidores e da organização fazendária. Suas principais atividades compreendem: coordenação do Planejamento Estratégico; coordenação do Programa de Comunicação Interna; coordenação do Programa de Qualidade de Vida; coordenação do Programa Socioambiental; e Pesquisas de Satisfação e de Clima Organizacional.

\section{Referências}

Aquino, A. C. B.; Cardoso, R. L.; Pagliarussi, M. S.; Boya, V. L. A. Causality in a performance measurement model: a case study in a Brazilian power distribution company. In: Epstein, M.J.; Manzoni, J. (Org.). Performance Measurement and Management Control: Measuring and rewarding performance - Studies in Managerial and Financial Accounting. - ed. Bingley: Emerald Group Publishing Limited, 18, 273-299, 2008.

Ammons, D. N.; Rivenbark, W. C. Factors influencing the use of performance data to improve municipal services: evidence from the North Carolina benchmarking project. Public Administration Review, 68(2), 2008.

Banco Central do Brasil. Página Oficial do Banco Central do Brasil. 2008. Disponível em: http://www4.bcb.gov.br/?Economia. Acesso em: 22 de fevereiro de 2008.

Besanko, D.; Dranove, D.; Shanley, M.; Schaefer, S. Economics of strategy. New York:Wiley, 2007. 
Bourdeaux, C. Do legislatures matter in budgetary reform? Public Budgeting \& Finance, 26(1), 120-142, 2006.

BROWNELL, P. The role of accounting data in performance evaluation, budgetary participation, and organizational effectiveness. Journal of Accounting Research, 20(1), 1982.

CAIDEn, N. Public service professionalism for performance measurement and evaluation. Public Budgeting \& Finance, 18, 35-52, 1998.

Epstein, M. J.; Manzoni, J. C. The balanced scorecard and tableau de bord: translating strategy into action. Management Accounting, 79, 28-36, 1997.

Fundação de Economia e Estatística (FEE). Governo do Estado do Rio Grande do Sul, Secretaria de Planejamento e Gestão. Estatísticas. Disponível em: http:// www.fee.rs.gov.br/sitefee/pt/content/estatisticas/pg_pib_estado_desempenho.php. Acesso em: 22 de fevereiro de 2008.

HaRTMAnN, F. G. H. The appropriateness of RAPM: toward the further development of theory. Accounting, Organizations and Society, 25, 451-482, 2000.

Humphrey, C.; Scapens, R. W. Theories and case studies of organizational accounting practices: limitation or liberation? Accounting, Auditing \& Accountability Journal, 9 (4), 86-106, 1996.

ItTner, C.; Larcker, D.; Meyer, M. Subjectivity and the weighting of performance measures: evidence from a balanced scorecard. The Accounting Review, 78 (3), 725-758; 2003.

JAMES, O. The UK core executive's use of public service agreements as a tool of governance. Public Administration, 82 (2), 397-419, 2004.

Jordan, M. Hackbart, M. Performance budgeting and performance funding in the States: a States assessment. Public Budgeting \& Finance, 19, 68-88, March 1999.

Kaplan, R.S.; NorTON, D.P. The balanced scorecard: translating strategy into action. Cambridge: Harvard Business School Press, 1996.

Malina, M. A; Selto, F. H. Communicating and controlling strategy: an empirical study of the effectiveness of the balanced scorecard. Journal of Management Accounting Research, 13, 441-469, 2001.

. Causality in a performance measurement model. Working paper. Acessado em: 25 de março de 2009. Disponível em: http://papers.ssrn.com/sol3/ papers.cfm?abstract_id=488144, 2004.

Melkers, J. E.; Willoughby, K. G. Models of performance-measurement use in local governments: Understanding budgeting, communication, and lasting effects. Public Administration Review, 65(2), 180-191, 2005.

Rio Grande do Sul (RS). Página Oficial do Governo do Rio Grande do Sul. Disponível em: http://www.rs.gov.br/. Acesso em: 22 de fev.2008.

Merchant, K. A. Budgeting and the propensity to create budget slack. Accounting, Organizations and Society, 10, 201-210, 1985. 
Paduan, R. A “banda” boa do setor público. Exame. São Paulo, n. 950, p.102-109, 2009.

Secretaria de Estado da Fazenda do Rio Grande do Sul (Sefaz). Portal de Legislação. Disponível em:http://www.legislacao.sefaz.rs.gov.br/Site/Document.aspx?inpKey= 99608\&inpCodDispositive=\&inpDsKeywords=. Acesso em: 22 de fev. 2008.

SCAPENS, R. Never mind the gap: towards an institutional perspective on management accounting practice. Management Accounting Research, v5, 1994.

Severo, A. M. V. Modelo de Gestão da Sefaz-RS. Conferência Para A Secretaria De Estado Da Fazenda Do Amazonas, 2006. Manaus, 2006.

Sprinkie, G. B. Perspectives on experimental research in managerial accounting. Accounting, Organizations and Society, 28, 287-318, 2003.

WEBB, A. Managers' commitment to the goals contained in a strategic performance measurement system. Contemporary Accounting Research, 11, 1-29, 2004.

Widener, S. Human capital, pay structure, and the use of performance measure in bonus compensation. Management Accounting Research, 17, 198-221, 2006

Willoughby, Katherine G. Performance measurement and budget balancing: State government perspective. Public Budgeting and Finance, 24(2), 21-39, June 2004. 


\section{Resumo-Resumen-Abstract}

\section{Mensuração de Desempenho em Organizações Públicas: o caso da Secretaria da Fazen-} da do Rio Grande do Sul

Vinicius Pimentel de Freitas, Ricardo Lopes Cardoso, André Carlos Busanelli de Aquino e Rômulo Campos dos Reis Júnior

Este artigo apresenta o caso do modelo de mensuração de desempenho existente na Secretaria de Estado da Fazenda do Rio Grande do Sul. Mediante abordagem exploratória e descritiva, baseada em entrevistas em profundidade, comparamos as similaridades entre o modelo vigente na Secretaria e as principais características citadas nos estudos desses modelos aplicados à iniciativa privada. Encontramos como semelhanças: dissociação entre recompensa e esforço; negociação de metas; e mecanismos sociais de punição. A análise das séries trimestrais dos indicadores de desempenho, na comparação meta/realizado entre 2005-2008 (14 trimestres, até junho/2008), fornece evidências iniciais de que os funcionários da Secretaria têm atingido em média $91,46 \%$ das metas. Isso sugere a existência de folga orçamentária e, portanto, de baixo incentivo ao desempenho.

Palavras-chave: mensuração de desempenho, incentivos, remuneração variável.

\section{Evaluación de Desempeño en Organizaciones Públicas: el caso de la Secretaria de Hacienda del Estado de Rio Grande do Sul}

Vinicius Pimentel de Freitas, Ricardo Lopes Cardoso, André Carlos Busanelli de Aquino y Romulo Campos dos Reis Júnior

Este artículo presenta el caso del modelo de evaluación de desempeño de la Secretaria de Hacienda de Rio Grande do Sul. Mediante un abordaje exploratorio y descriptivo, basado en entrevistas en profundidad, comparamos las similitudes entre el modelo adoptado por la Secretaria y las características de los modelos adoptados por las empresas. Ejemplos de esas similitudes son: disociación entre el esfuerzo y la recompensa; negociación de metas; puniciones sociales. El análisis de las series trimestrales de los indicadores de desempeño, en la comparación meta/realizado entre 2005-2008 (14 trimestres, hasta juño/2008), provee evidencias iniciales de que los funcionarios han alcanzado en promedio un $91,46 \%$ de las metas. Esto sugiere la existencia de holgura presupuestaria; por lo tanto, un bajo incentivo al desempeño.

Palabras clave: evaluación de desempeño, incentivos, remuneración variable.

Performance Measurement in Public Organizations: the case of the Treasury Secretariat of Rio Grande do Sul State

Vinicius Pimentel de Freitas, Ricardo Lopes Cardoso, André Carlos Busanelli de Aquino and Romulo Campos dos Reis Júnior

This paper presents a case of a performance measurement model at the Treasury Secretariat of Rio Grande do Sul State. Based on an exploratory and descriptive approach, through in-depth interviews, we compared similarities between the model adopted at the Secretariat and the main features of those models applied by private organizations. Those similarities include: efforts and benefits dissociation; negotiation of thresholds; and social punishment. Analyzes of quarterly performance measurement indicators series, based on threshold/effective comparison along 20052008 (14 quarters, until June/2008), provide evidence that Secretariat's employees have reached on average $91.46 \%$ of Secretariat's thresholds. It suggests the presence of budgetary slack and so, low performance incentive.

Keywords: performance measurement, incentives, bonus. 
Vinicius Pimentel de Freitas

Mestrando, Fundação Getúlio Vargas, Escola Brasileira de Administração Pública e de Empresas (FGV/EBAPE) e Secretaria da Fazenda do Rio Grande do Sul (Sefaz/RS). Contato: vinicius.freitas@sefaz.rs.gov.br

Ricardo Lopes Cardoso

Doutor, Fundação Getúlio Vargas, Escola Brasileira de Administração Pública e de Empresas (FGV/EBAPE). Contato: ricardo.lopes.cardoso@fgv.br

André Carlos Busanelli de Aquino

Doutor, Universidade de São Paulo, Faculdade de Economia, Administração e Contabilidade de Ribeirão Preto (USP/FEARP). Contato: aaquino@usp.br

Romulo Campos dos Reis Júnior

Mestrando, Universidade de São Paulo, Faculdade de Economia, Administração e Contabilidade de Ribeirão Preto (USP/FEARP). Contato: romulocri@gmail.com 